\title{
Adaptive $M$-PSK Communications in the Absence of Channel Gain Estimation
}

\author{
Athanasios S. Lioumpas ${ }^{1}$, George K. Karagiannidis ${ }^{1}$ and Diomidis S. Michalopoulos ${ }^{2}$ \\ ${ }^{1}$ Department of Electrical and Computer Engineering, Aristotle University of Thessaloniki, Thessaloniki, Greece \\ Email: \{alioumpa, geokarag\}@ auth.gr \\ ${ }^{2}$ Department of Electrical and Computer Engineering, The University of British Columbia, Vancouver, Canada \\ Email: dio@ece.ubc.ca
}

\begin{abstract}
We introduce a variable-rate (VR) $M$-PSK modulation scheme, for communications over fading channels, in the absence of channel gain estimation at the receiver. The choice of the constellation size is based on the signal-plus-noise $(S+N)$ sampling value rather than on the signal-to-noise ratio $(S / N)$. It is analytically shown that $S+N$ can serve as an excellent simpler criterion, alternative to $S / N$, for determining the modulation order in $V R$ systems. In this way, low complexity transceivers can use $V R$ transmissions in order to increase their spectral efficiency under an error performance constraint.
\end{abstract}

\section{INTRODUCTION}

A common technique for coping with fading in wireless communications, is transmission or reception diversity, which provides performance improvement at the cost of extra hardware or increased bandwidth usage. Alternatively, if a feedback link is available, the fading can be mitigated by allowing the receiver to monitor the channel conditions and request compensatory changes in certain parameters of the transmitted signal. This technique is called adaptive transmission and its basic concept is the real-time balancing of the link budget through adaptive variation of the transmitted power level, symbol transmission rate, constellation size, coding rate/scheme, or any combination of these parameters.

Adapting to the signal fading allows the channel to be used more efficiently since power and rate can be allocated to take advantage of favorable channel conditions. Adaptive transmission was first proposed by Hayes [1], who considered a Rayleigh fading channel where the amplitude of the transmitted signal is under control of the receiver through a feedback channel.

\section{A. Motivation}

Several works have dealt with adaptive transmission systems, in which the modulation/coding parameters, such as constellation size and coding rate are adaptively varied with the prevailing fading channel condition while maintaining a certain error rate performance [2]-[11]. These techniques have been also considered in several modern terrestrial and satellite communication systems, also incorporated in standards (e.g. High-Speed Downlink Packet Access - HSDPA [12] and Digital Video Broadcasting - Return Channel via Satellite DVB-RCS [13]).
In many practical applications, the adaptive transmission is limited to variable-rate $(V R)$ schemes, since the difference from variable-power $(V P) V R$, is a small fraction of decibel for most types of fading [4]. Most of the $V R$ communication systems proposed in the literature, adapt the transmission parameters according to the instantaneous channel condition, limiting their applicability to systems that estimate the instantaneous channel gain, either perfectly or with estimation errors.

\section{B. Previous Works and Contribution}

To the best of the authors' knowledge, no $V R$ modulation scheme has been proposed in the literature that does not require channel gain estimation. In [14], the performance of a blind adaptive modulation scheme is investigated, which necessitates channel knowledge at the receiver but not at the transmitter. In this scheme, the order of an $M$-ary quadrature amplitude modulation ( $M$-QAM) sequentially decreases or increases according to positive/negative acknowledgments (ACK/NACK) sent to the transmitter.

In this paper, we propose a rate adaptation technique, which is based on the signal-plus-noise $(S+N)$ samples, instead of the instantaneous $S / N$. Owing to this particularity, no channel gain estimation is necessary for determining the transmission rate.

The $S+N$ technique has been applied to selection diversity communication systems (e.g. see [15] and the references therein), where its main advantage lies in taking into account the instantaneous noise power, in contrast to $S / N$ (e.g. the channel gain divided by the average noise power). The main contribution of this paper can be summarized as follows.

- We analytically show that $S+N$ can serve as an excellent alternative criterion to $S / N$, in $V R$ systems; the same spectral efficiency can be achieved, when either $S / N$ or $S+N$ is used for determining the constellation size.

- Based on this important outcome, we introduce an $S+N$ based $V R M$-ary phase shift keying ( $M$-PSK) modulation scheme that does not require any channel gain estimation. Note, that $M$-PSK modulation schemes have some important advantages over QAM schemes, e.g. improved performance when used with high power amplifiers (HPA). Another advantage of using $M$-PSK instead of $M$-QAM for Othogonal Frequency-Division Multiplexing (OFDM) systems is the availability of techniques to reduce the 
signal's Peak-to-Average-power Ratio (PAR), also known as PAPR or PMEPR (Peak-to-Mean Envelope Power Ratio) [16].

\section{Outline}

The remainder of the paper is organized as follows. In Section II, the $S+N$ based adaptation scheme is presented and compared with the $S / N$ one. Two criteria are applied for evaluating the efficiency of the $S+N$ scheme. The $V R M$-PSK modulation scheme is presented in Section III, along with numerical results. Concluding remarks are given in Section IV.

\section{II. $V R$ SYSTEMS WITH THE $S+N$ CRITERION}

We consider a discrete-time channel with stationary and ergodic time-varying gain $a[i]$. Assume that the fading amplitude $a[i]$ follows a Rayleigh distribution with probability density function (pdf)

$$
f_{a}(a)=\frac{2 a}{\Omega} e^{-\frac{a^{2}}{\Omega}},
$$

where $\Omega=E\left\{a^{2}\right\}$. The average channel gain is denoted as $\bar{\gamma}$, which is assumed to be $\bar{\gamma}=1$, without loss of the generality. The instantaneous received signal is

$$
r[i]=a[i] e^{j \vartheta[i]} s[i]+n[i],
$$

where $s[i]$ is the complex transmitted symbol, $\vartheta[i]$ is the phase introduced by the fading channel and $n[i]$ is a zero mean circularly symmetric complex Gaussian noise with $E\left\{n[i]^{*} n[i]\right\}=N_{0}=2 \sigma^{2}$. The symbol $s[i]$ is selected from a signal constellation with $M$ symbols (e.g. $M$-QAM or $M$ PSK) each with energy $E_{S_{k}}$ and total average symbol energy $E_{S}=1$. Then, the received $S / N$ will be

$$
\gamma[i]=E_{S} \frac{a^{2}[i]}{B N_{0}},
$$

where $B$ is the bandwidth of the received signal.

In conventional $V R$ systems, the modulation order used at the time instant $i+1$, is determined by the value of the received $S / N, \gamma[i]$, at the time instant $i$, and a predetermined symbol or bit error rate (SER or BER) target. The objective is to maximize the spectral efficiency by using the largest possible constellation size for transmission under the instantaneous error rate requirement. Towards this aim, a mathematical formulation that relates the SER, $P_{S}$, with the modulation order, $M$, and the received $S / N, \gamma[i]$, are necessary. These formulas must be simple enough, so that $M$ can be directly expressed as a function of $P_{S}$ and $\gamma[i]$, i.e., $M=f\left(\gamma[i], P_{S}\right)$. In the following, the time notation, $i$, will be omitted.

Given the direct relation between $M$ and $\gamma$, at each symbol time, the constellation size, $M$, is selected from $N$ available orders, i.e., $\left\{M_{1}, M_{2}, M_{3}, M_{4}, \ldots, M_{N}\right\}$, depending on which region $M=f\left(\gamma, P_{S}\right)$ lies; if $M_{j} \leq M<M_{j+1}$, $j=1, \ldots, N$, then the modulation order will be equal to
$M_{j}$. Because of the relation between $M, \gamma$ and $P_{S}$, the constellation size can be equivalently determined by $\gamma$, after discretizing the range of channel fade levels. Specifically, the range of $\gamma$ is divided into $N+1$ fading regions $\left[\gamma_{j}, \gamma_{j+1}\right]$, and the constellation $M_{j}$ is associated with the $j$ th region.

In summary, the modulation order is determined as

$$
M=M_{j}, \quad \text { if }\left\{\begin{array}{c}
M_{j} \leq M<M_{j+1} \\
o r \\
\gamma_{j} \leq \gamma<\gamma_{j+1} \Leftrightarrow \gamma_{j} \leq E_{S_{k}} \frac{a^{2}}{N_{0}}<\gamma_{j+1},
\end{array}\right.
$$

where $\gamma_{j} \mathrm{~s}$ are calculated from $M=f\left(\gamma, P_{S}\right)$.

For example, assuming a $V R M$-QAM scheme, a mathematical expression for associating $M, \gamma$ and $P_{S}$ may be the following [17, Ch. 7]

$$
P_{s} \leq 2 \operatorname{erfc}\left(\sqrt{\frac{3 \gamma}{2(M-1)}}\right),
$$

with $\operatorname{erfc}(\cdot)$ denoting the complementary error function. (5) can be solved with respect to $M$, to obtain the maximum modulation order as a function of the instantaneous $S / N$, as

$$
M=1+\frac{3 \gamma}{\left(\sqrt{2} \operatorname{erfc}^{-1}\left(P_{s} / 2\right)\right)^{2}},
$$

where $\operatorname{erfc}^{-1}(\cdot)$ stands for the inverse complementary error function. Finally, from (6), the fading regions are obtained as

$$
\gamma_{j}=\frac{\left(M_{j}-1\right)}{3}\left(\sqrt{2} \operatorname{erfc}^{-1}\left(P_{s} / 2\right)\right)^{2} .
$$

In this paper, we propose an alternative criterion for determining the modulation order in $V R$ systems, which does not require the estimation of the fading amplitude and is only based on the scaled $S+N$ sample as

$$
\xi[i]=\frac{\Re\left\{r[i] e^{-j \vartheta[i]}\right\}^{2}}{N_{0}}=\frac{\left(a[i] s[i]+n_{I}[i]\right)^{2}}{N_{0}}
$$

where $\Re\{z\}$ denotes the real part of $z$ and $n_{I}[i]$ is the in-phase noise component with variance $N_{0} / 2$.

In the proposed $S+N$ scheme, the modulation order is chosen similarly to the conventional one, with the difference that $\gamma$ is replaced by $\xi$, i.e. the selected order, $M$, will be

$$
M=M_{j}, \quad \text { if }\left\{\begin{array}{c}
\gamma_{j} \leq \xi<\gamma_{j+1} \\
o r \\
\gamma_{j} \leq \frac{\left(a \sqrt{E_{S_{k}}}+n_{I}\right)^{2}}{N_{0}}<\gamma_{j+1},
\end{array}\right.
$$

Assuming constant energy transmissions, the effectiveness of the $S+N$ scheme compared to the $S / N$ can be verified via the following criteria.

Criterion 1: The probability that the same constellation size is determined by both $S / N$ and $S+N$, i.e.,

$$
\begin{aligned}
\Pi_{1}= & \operatorname{Pr}\left\{\gamma_{j} \leq E_{S} \frac{a^{2}}{N_{0}}<\gamma_{j+1} \cap\right. \\
& \left.\gamma_{j} \leq \frac{\left(a \sqrt{E_{S}}+n_{I}\right)^{2}}{N_{0}}<\gamma_{j+1}\right\} .
\end{aligned}
$$




$$
\Pi_{1}=\operatorname{Pr}\left\{\sqrt{N_{0} \gamma_{j}} \leq y<\sqrt{N_{0} \gamma_{j+1}} \cap \sqrt{N_{0} \gamma_{j}}-y \leq n_{I}<\sqrt{N_{0} \gamma_{j+1}}-y\right\}
$$

Equivalently, (10) can be re-written as in (11) at the top of the page, where $y=a \sqrt{E_{S}}$ is a random variable with a pdf calculated as follows, after applying the transformation [19, (5.6)],

$$
f_{y}(y)=\frac{1}{\sqrt{E_{S}}} f_{a}\left(\frac{y}{\sqrt{E_{S}}}\right)=\frac{2 y}{\Omega E_{s}} e^{-\frac{y^{2}}{\Omega E_{s}}} .
$$

Using the cumulative distribution function (cdf) of the zeromean Gaussian random variable, $x$, $[18,($ A.18)]

$$
F_{X}(x)=\int_{-\infty}^{x} \frac{1}{\sqrt{2 \pi \sigma}} e^{-\frac{z^{2}}{2 \sigma^{2}}} d z
$$

and the definition of the complementary error function, [18, (A.24)]

$$
\operatorname{erfc}(x)=\frac{2}{\sqrt{\pi}} \int_{x}^{\infty} e^{-z^{2}} d z
$$

the probability

$$
\operatorname{Pr}\left\{\sqrt{N_{0} \gamma_{j}}-y \leq n_{I}<\sqrt{N_{0} \gamma_{j+1}}-y\right\}
$$

can be calculated after some trivial manipulations as

$$
\begin{aligned}
& \operatorname{Pr}\left\{\sqrt{N_{0} \gamma_{j}}-y \leq n_{I}<\sqrt{N_{0} \gamma_{j+1}}-y\right\} \\
= & \frac{1}{2}\left[\operatorname{erfc}\left(\frac{\sqrt{N_{0} \gamma_{j}}-y}{\sqrt{N_{0}}}\right)-\operatorname{erfc}\left(\frac{\sqrt{N_{0} \gamma_{j+1}}-y}{N_{0}}\right)\right] .
\end{aligned}
$$

Thus, the probability, $\Pi_{1}$, can be written as in (14) at the top of the next page.

Now, in order to derive a closed-form expression for (14), we have to solve integrals of the form

$$
I=D \int_{t}^{y} \operatorname{erfc}\left(\frac{E-x}{\sqrt{2} C}\right) x e^{-D x^{2}} d x .
$$

Fortunately, a closed-form solution to (15) can be found in [20, Vol. 2, (1.5.3.10)] as

$$
\begin{gathered}
I=g(t, y, z, w, v)= \\
\frac{1}{2}\left\{e^{-t^{2} w}-2 e^{-y^{2} w}+e^{-t^{2} w}\left[1-\operatorname{erfc}\left(\frac{t-v}{\sqrt{2} z}\right)\right]+\right. \\
e^{-y^{2} w} \operatorname{erfc}\left(\frac{y-v}{\sqrt{2} z}\right)+\frac{e^{-\frac{w v^{2}}{1+2 z^{2} w}}}{\sqrt{1+2 z^{2} w}} \\
{\left[\operatorname{erfc}\left(\frac{t+2 t z^{2} w-v}{z \sqrt{2+4 z^{2} w}}\right)-\operatorname{erfc}\left(\frac{y+2 y z^{2} w-v}{z \sqrt{2+4 z^{2} w}}\right)\right],}
\end{gathered}
$$

Finally, combining (16) and (14) a closed-form solution to $\Pi_{1}$, is derived as in (17), at the top of the next page.
Criterion 2: The probability that the constellation size, determined by $S+N$, is $M$, given that the constellation size, determined by $S / N$ is also $M$, i.e.,

$$
\begin{aligned}
& \Pi_{2}=\operatorname{Pr}\left\{\gamma_{j} \leq \frac{\left(a \sqrt{E_{S}}+n_{I}\right)^{2}}{N_{0}}<\gamma_{j+1}\right. \\
& \left.\mid \gamma_{j} \leq E_{S} \frac{a^{2}}{N_{0}}<\gamma_{j+1}\right\} .
\end{aligned}
$$

The probability in (18), can be calculated as in (14), but now, the random variable, $y$, is limited inside the interval $\left[\sqrt{N_{0} \gamma_{j}}, \sqrt{N_{0} \gamma_{j+1}}\right]$. This is implied by the fact that the probability

$$
\operatorname{Pr}\left\{\gamma_{j} \leq \frac{\left(a \sqrt{E_{S}}+n_{I}\right)^{2}}{N_{0}}<\gamma_{j+1}\right\}
$$

is conditioned on the event

$$
\operatorname{Pr}\left\{y \in\left[\sqrt{N_{0} \gamma_{j}}, \sqrt{N_{0} \gamma_{j+1}}\right]\right\}=1
$$

An approximate solution can be obtained by "forcing" the pdf, $f_{y}(y)$, to have unitary integral when integrated from $\sqrt{N_{0} \gamma_{j}}$ to $\sqrt{N_{0} \gamma_{j+1}}$. In other words, we have to average the probability

$$
\operatorname{Pr}\left\{\sqrt{N_{0} \gamma_{j}}-y \leq n_{I}<\sqrt{N_{0} \gamma_{j+1}}-y\right\}
$$

in (14) over a new pdf, $h_{y}(y)$, defined as

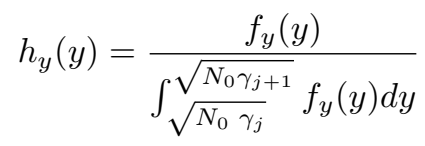

so that

$$
\int_{\sqrt{N_{0} \gamma_{j}}}^{\sqrt{N_{0} \gamma_{j+1}}} h_{y}(y) d y=1 .
$$

Therefore, (18) can be approximated by

$$
\Pi_{2} \simeq \Pi_{1} \frac{h_{y}(y)}{f_{y}(y)} .
$$

Numerical results considering these criteria will be presented in the next section, where the $S+N$ scheme is applied in a $V R M$-PSK modulation scheme.

\section{VARIABLE RATE $M$-PSK}

\section{A. Mode of Operation}

In this section, we introduce a constant power, $V R M$-PSK modulation scheme, in which the decision on the modulation order is based on the $S+N$ samples rather than on the $S / N$ ones. The mode of operation is illustrated in Fig. 1. An ideal 


$$
\begin{aligned}
\Pi_{1} & =\int_{\sqrt{A_{N} N_{0}}}^{\sqrt{A_{N+1} N_{0}}} \operatorname{Pr}\left\{\sqrt{N_{0} \gamma_{j}}-y \leq n_{I} i \sqrt{N_{0} \gamma_{j+1}}-y\right\} f_{y}(y) d y= \\
& =\frac{1}{2} \int_{\sqrt{N_{0} \gamma_{j}}}^{\sqrt{N_{0} \gamma_{j+1}}}\left[\operatorname{erfc}\left(\frac{\sqrt{N_{0} \gamma_{j}}-y}{\sqrt{N_{0}}}\right)-\operatorname{erfc}\left(\frac{\sqrt{N_{0} \gamma_{j+1}}-y}{N_{0}}\right)\right] f_{y}(y) d y
\end{aligned}
$$

$$
\Pi_{1}=g\left(\sqrt{N_{0} \gamma_{j}}, \sqrt{N_{0} \gamma_{j+1}}, \sqrt{\frac{N_{0}}{2}}, \frac{1}{E s \Omega}, \sqrt{N_{0} \gamma_{j}}\right)-g\left(\sqrt{N_{0} \gamma_{j}}, \sqrt{N_{0} \gamma_{j+1}}, \sqrt{\frac{N_{0}}{2}}, \frac{1}{E s \Omega}, \sqrt{N_{0} \gamma_{j+1}}\right)
$$

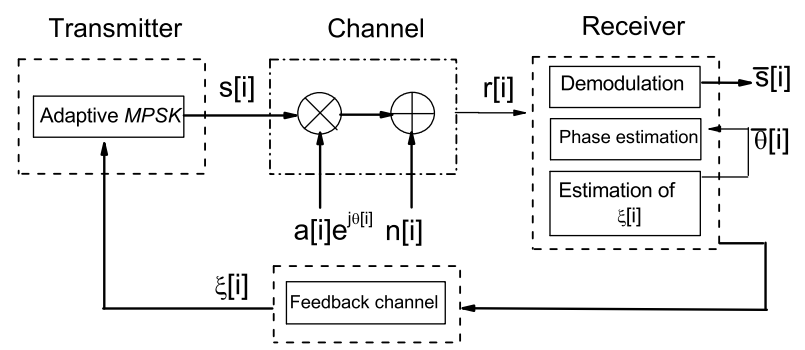

Fig. 1. The VR M-PSK modulation scheme.

coherent phase detection is assumed to be available at the receiver at time instant, $i$, while no channel gain estimation is necessary. The receiver estimates the $S+N$ samples at time, $i$, and decides on the constellation size $M_{j}=2^{j}, j=1$, $2, \ldots, N$, that will be used at the next time interval, $i+1$, according to this sample. The decision on the modulation order is sent back to the transmitter, transmitting $N$ bits through a feedback channel, that does not introduce any errors, which can be assured by increasing its delay time and using an ARQ transmission protocol.

As mentioned, the estimation of the modulation order requires a closed-form formula that relates, the $S / N$ with the error probability. For the case of the $M$-PSK we can use the approximation for the SER over AWGN, i.e., [17, Ch. 7]

$$
P_{M} \approx \operatorname{erfc}\left(\sqrt{\gamma} \sin \frac{\pi}{M}\right)
$$

Solving (23) with respect to $M$, the maximum constellation size for a given SER is given by

$$
M=\frac{\pi}{\arcsin \left(\frac{1}{\gamma} \operatorname{erfc}^{-1} P_{M}\right)} .
$$

Therefore, the selected order, $M$, will be

$$
M=M_{j}, \quad \text { if }\left\{\begin{array}{c}
M_{j} \leq M<M_{j+1} \\
o r \\
\gamma_{j} \leq \frac{\left(a \sqrt{E_{S}}+n_{I}\right)^{2}}{N_{0}}<\gamma_{j+1}
\end{array},\right.
$$

where

$$
\gamma_{j}=\frac{\operatorname{erfc}^{-1} P_{M}}{\sin \frac{\pi}{M_{j}}} .
$$

\section{B. Performance Evaluation}

1) Spectral efficiency: The normalized spectral efficiency of the $S+N$ based $V R M$-PSK scheme is obtained as

$$
\frac{R}{B}=\sum_{j=1}^{N} \log _{2}\left(M_{j}\right) \operatorname{Pr}\left\{\gamma_{j} \leq \xi<\gamma_{j+1}\right\} .
$$

The probability, $\operatorname{Pr}\left\{\gamma_{j} \leq \xi<\gamma_{j+1}\right\}$, in (27) can be rewritten as

$$
\begin{aligned}
& \operatorname{Pr}\left\{\gamma_{j} \leq \xi<\gamma_{j+1}\right\} \\
= & \operatorname{Pr}\left\{\sqrt{\gamma_{j} N_{0}} \leq a \sqrt{E_{S}}+n_{I}<\sqrt{\gamma_{j+1} N_{0}}\right\} .
\end{aligned}
$$

The random variable $z=a \sqrt{E_{S}}+n_{I}$, which is the sum of a Rayleigh and a Gaussian random variables, has the following pdf (the proof is included in the journal version of this work)

$$
\begin{gathered}
f_{z}(z)=\frac{1}{\sqrt{2 \pi}\left(2 \sigma^{2}+E_{s} \Omega\right)^{1.5}}\left\{e^{-\frac{1}{2}\left(\frac{1}{\sigma^{2}}+\frac{1}{E_{s} \Omega}\right) z^{2}}\right. \\
{\left[2 e^{\frac{z^{2}}{E_{s} \Omega}} \sigma \sqrt{2 \sigma^{2}+E_{s} \Omega}+e^{\frac{\left(4 \sigma^{2}+2 \sigma^{2} E_{s} \Omega+E_{s}^{2} \Omega^{2}\right)^{2}}{2 \sigma^{2} E_{s} \Omega\left(2 \sigma^{2}+E_{s} \Omega\right)}}\right.} \\
\left.\left.\sqrt{2 \pi E_{s} \Omega}\left(1+\operatorname{erf}\left(\frac{E_{s} \Omega}{\sqrt{2 \sigma^{2} E_{s} \Omega\left(2 \sigma^{2}+E_{s} \Omega\right)}} z\right)\right)\right]\right\} .
\end{gathered}
$$


As a result,

$$
\operatorname{Pr}\left\{\gamma_{j} \leq \xi<\gamma_{j+1}\right\}=\int_{\gamma_{j}}^{\gamma_{j+1}} f_{z}(z) d z
$$

which can be replaced in (27), to obtain the spectral efficiency of the $S+N$ based $V R M$-PSK scheme.

On the other hand, the normalized capacityspectral efficiency of a $V R M$-PSK scheme, with the modulation order determined by the received $S / N$, is given by

$$
\frac{R}{B}=\sum_{j=1}^{N} \log _{2}\left(M_{j}\right) \operatorname{Pr}\left\{\gamma_{j} \leq \gamma<\gamma_{j+1}\right\} .
$$

For the case that the fading amplitude $a$ follows a Rayleigh distribution, $\gamma$ will be exponentially distributed as

$$
f(\gamma)=\frac{1}{\bar{\gamma}} e^{-\frac{\gamma}{\gamma}}
$$

where $\bar{\gamma}=\Omega E_{s} / N_{0}$ and thus

$$
\operatorname{Pr}\left\{\gamma_{j} \leq \gamma<\gamma_{j+1}\right\}=\int_{\gamma_{j}}^{\gamma_{j+1}} \frac{1}{\bar{\gamma}} e^{-\frac{\gamma}{\gamma}} d \gamma=e^{-\frac{\gamma_{j}}{\bar{\gamma}}}-e^{-\frac{\gamma_{j+1}}{\bar{\gamma}}} .
$$

2) Symbol Error Probability: Regarding the SER of a $S / N$ based $V R M$-PSK scheme, it can be obtained by averaging over the SER for $M$-PSK in AWGN [5], i.e.,

$$
P_{s}=\sum_{j} \int_{\gamma_{j}}^{\gamma_{j+1}} P_{A W G N}\left(M_{j}, \gamma\right) f_{\gamma}(\gamma) d \gamma
$$

The SER of $M$-PSK over AWGN is given by [21, Ch. 8.1]

$$
\begin{aligned}
& P_{A W G N}\left(M_{j}, \gamma\right)=Q(\sqrt{2 \gamma})+ \\
& \frac{2}{\sqrt{\pi}} \int_{0}^{\infty} \exp \left\{-(u-\sqrt{\gamma})^{2}\right\} Q\left(\sqrt{2} \gamma \tan \frac{\pi}{M}\right) d u
\end{aligned}
$$

The above approach results in some cases to closed-form solutions for the error probability.

For the case of the $S+N$, however, a similar approach cannot be followed, since a formula for the SER as a function of $\xi$ is required. Moreover, when physically realizing $S+N$ schemes, though, by sampling the output of a matched filter, the noise is a random variable. Thus, it is inexact to specify the performance of $S+N$ systems using a constant noise analysis [22]. Because of the above reasons, the SER for the proposed scheme is calculated via computer simulations.

\section{Results and Discussion}

The evaluation of the probabilities, $\Pi_{1}$ and $\Pi_{2}$ is very important, since they imply the efficiency of $S+N$ to estimate the quality of the instantaneous received signal. For the case of the $M$-PSK, $\Pi_{1}$ and $\Pi_{2}$ are plotted in Figs. 2 and 3 respectively. Note, that the simulation results coincide perfectly with the analytical ones, for both these probabilities.

A general conclusion derived form these figures is that if the SNR based $V R M$-PSK uses a specific modulation order, the probability that the $S+N$ based $V R M$-PSK uses the same

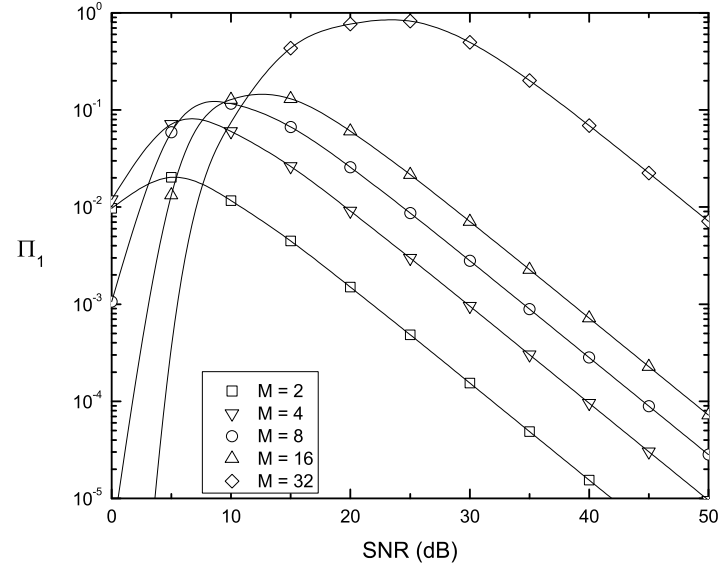

Fig. 2. The probability, $\Pi_{1}$, for a $V R$ M-PSK modulation scheme. Theoretical (solid lines) and simulation (symbols) results.



Fig. 3. The probability, $\Pi_{2}$, for a $V R$ M-PSK modulation scheme. Theoretical (solid lines) and simulation (symbols) results.

order is relatively high. Moreover, as expected, the efficiency of the $S+N$ improves as the average $S / N$ increases, since the instantaneous noise components become small, so that $\left(a \sqrt{E_{S}}+n\right)^{2} / N_{0}$ and $a^{2} E_{S} / N_{0}$ become almost the same.

While the proposed $V R M$-PSK may not use momentarily the same constellation size as that of the conventional one, it does necessarily mean that it will lead to inferior error performance. On the contrary, as shown in Fig. 5, the SER of the $S+N V R M$-PSK is the same as that of the $S / N V R M$ PSK, while they achieve the same spectral efficiency (Fig. 4). In other words, it can be concluded that the channel quality can be estimated with $S+N$ as reliably as via the $S / N$.

The explanation for the efficiency of the $S+N$ criterion is as follows: in the conventional $V R M$-PSK, the decision 


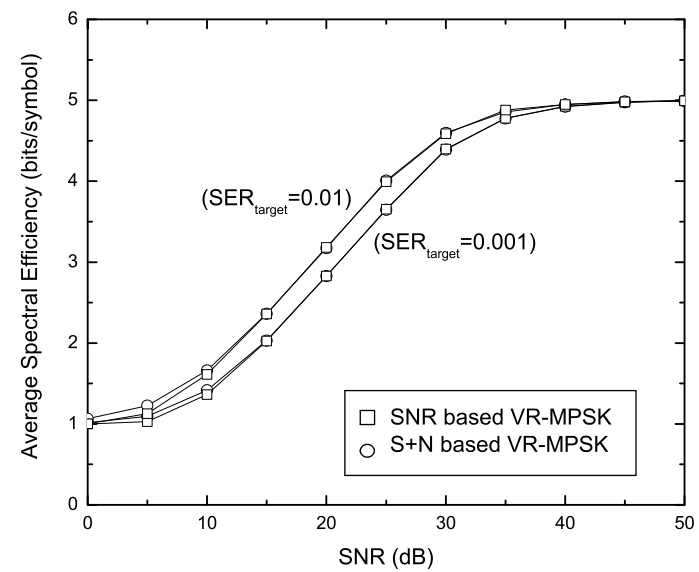

Fig. 4. The spectral efficiency of $V R$ M-PSK schemes, for different SER targets.

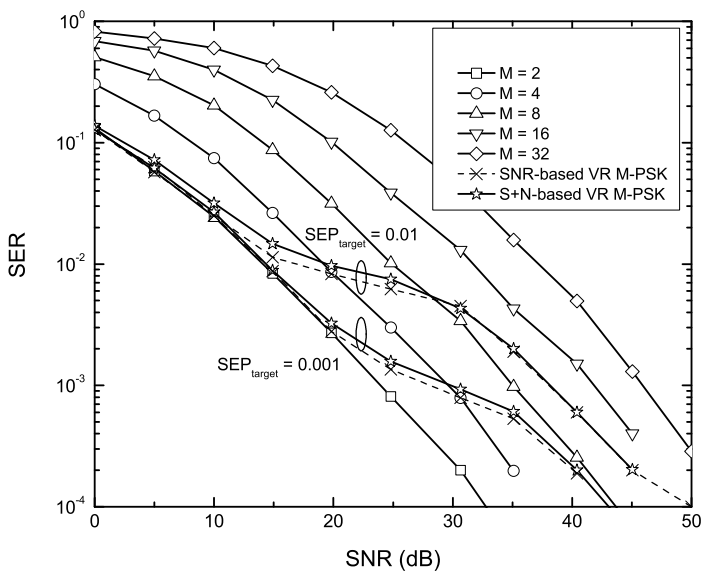

Fig. 5. The SER of $V R$ M-PSK schemes, for different SER targets.

on the modulation order is based on the instantaneous $S / N$, i.e. on $a^{2} E_{S} / N_{0}$, which means that the instantaneous noise power is ignored, since only the average noise power is taken into account, in contrast to $S+N$, which includes also the instantaneous noise component.

\section{CONCLUSions}

A VR $M$-PSK modulation scheme was introduced for wireless communication systems with no fading amplitude estimation capabilities. The choice of the constellation size is based on the $S+N$ samples, rather than on the $S / N$. It was analytically shown that the $S+N$ criterion is an attractive alternative to $S / N$ for choosing the appropriate modulation order in $V R$ communication systems. Towards this concept, a $V R M$-PSK modulation scheme was proposed, which requires no channel gain estimation and increases their spectral efficiency of $M$ PSK systems under the instantaneous error rate requirement.

\section{REFERENCES}

[1] J. F. Hayes, "Adaptive feedback communications," IEEE Trans. Commun. Technol., vol. COM-16, pp. 29-34, Feb. 1968.

[2] G. J. Clowes, "Variable rate data transmission for a Rayleigh fading channel," Commun. Lab., Defence Res. Telecommun. Establishment, Ottawa, Ont., Canada, Tech. Memo. 22, Feb. 1969.

[3] A. Goldsmith and P. Varaiya, "Capacity of Fading Channels with Channel Side Information", IEEE Trans. on Information Theory, Vol. IT-43, pp. 1896-1992, 1997.

[4] A. J. Goldsmith and S. G. Chua, "Variable-rate variable-power $M$-QAM for fading channels," IEEE Trans. Commun., vol. 45, no. 10, pp. 12181230, Oct. 1997.

[5] M-S. Alouini and A. J. Goldsmith, "Adaptive modulation over Nakagami fading channels," Kluwer Wireless Personal Commun., vol. 13, pp. 119143, May 2000.

[6] X. Qiu and K. Chawla, "On the performance of adaptive modulation in cellular systems," IEEE Trans. Commun., vol. 47, no. 6, pp. 884-895, June 1999.

[7] R. K. Mallik, M. Z. Win, J. W. Shao, M. S. Alouini, and A. J. Goldsmith, "Channel capacity of adaptive transmission with maximal ratio combining in correlated Rayleigh fading," IEEE Trans. Wireless Commun., vol. 3, no. 4, pp. 1124-1133, July 2004.

[8] Q. Liu, S. Zhou, and G. B. Giannakis, "Cross-Layer Combining of Adaptive Modulation and Coding with Truncated ARQ Over Wireless Links," IEEE Trans. Wireless Commun., vol. 3, no. 5, Sept. 2004, pp. $1746-55$.

[9] M. Filip and E. Vilar, "Optimum utilization of the channel capacity of a satellite link in the presence of amplitude scintillations and rain attenuation," IEEE Trans. Commun., vol. 38, pp. 1958-1965, Nov. 1990.

[10] M. Filip and E. Vilar, "Implementation of adaptive modulation as a fade countermeasure," Int. J. Satellite Commun., vol. 12, pp. 181-191, 1994.

[11] Y-C. Ko, H-C Yang, S-S. Eom, and M-S. Alouini, "Adaptive Modulation with Diversity Combining Based on Output-Threshold MRC," IEEE Trans. Wireless Commun., vol. 6, no. 10, pp. 3728-3737, Oct. 2007.

[12] 3GPP TS 25.308 V5.2.0 (2002-03), "HSDPA Overall Description", (Rel.5).

[13] ETSI EN 301 790, v. 1.3.1. Digital video broadcasting (DVB); interaction channel for satellite distribution systems. http://www.etsi.org

[14] Y. Al-Harthi, A. H. Tewfik, M-S. Alouini, "Blind adaptive modulation systems for wireless channels with binary feedback," Wireless Communications and Mobile Computing, vol. 7, no. 3, pp. 257-266, Mar. 2006.

[15] Y. G. Kim, and N. C. Beaulieu, "Lossless Selection Combining without Channel State Information," IEEE International Conference on Communications, 2008. ICC '08, Beijing, pp. 1328-1332, May 2008.

[16] A. G. Armada, "SNR gap approximation for $M$-PSK-based bit loading," IEEE Transactions on Wireless Communications, vol. 5, no. 1, pp. 5760, 2006.

[17] J.G. Proakis and M. Salehi, Communication Systems Engineering, 2nd ed., Prentice Hall, 2002, pp. 615-623,586-588.

[18] G. L. Stüber, Principles of Mobile Communications. Norwell, MA: Kluwer Academic Publishers, 1996.

[19] A. Papoulis, Probability, Random Variables, and Stochastic Procceses, 3rd ed. McGraw-Hill, 1991.

[20] Prudnikov AP, Brychkov YA, Marichev OI. Integrals and Series. Gordon and Breach: New York, 1986.

[21] M. K. Simon and M. Alouini, Digital Communication over Fading Channels, 2nd ed. New Jersey: John Wiley, 2005.

[22] E. A. Neasmith and N. C. Beaulieu, "New results on selection diversity," IEEE Trans. Commun., vol. 46, No. 5, pp. 695-704,May 1998. 